\title{
Single Fusion Events at Polarized Liquid|Liquid Interfaces
}

\author{
Eduardo Laborda*[a], Angela Molina ${ }^{[a]}$, Vanesa Fernández Espín ${ }^{[a]}$, Francisco Martínez-Ortiz ${ }^{[a]}$, \\ José García de la Torre ${ }^{[a]}$, Richard G. Compton ${ }^{[b]}$
}

\begin{abstract}
We report a new electrochemical framework for tracking individual soft particles in solution and monitoring their fusion with polarized liquid|liquid interfaces. The physicochemical principle lies in the interfacial transfer of an ionic probe confined in the particles dispersed in solution and that is released upon their collision and fusion with the fluid interface. As a proof-of-concept, spike-like transients of a stochastic nature are reported in the current-time response of 1,2-dichloroethane(DCE)|water(W) submilli-interfaces after injection of DCE-in-W emulsions. The sign and potentialdependence of the spikes reflect the charge and lipophilicity of the ionic load of the droplets. Comparison with dynamic light scattering measurements indicates that each spike is associated with the collision of a single sub-picoliter droplet. This opens a new framework for the study of single fusion events at the micro- and nano-scales and of ion transport across biomimetic soft interfaces.
\end{abstract}

This work introduces a new framework, with roots in biomimetic liquid|liquid electrochemistry, for the study of single fusion events via impact experiments. ${ }^{[1,2]}$ Such events and associated ion transfers underpin much biology and diverse industrial processes. $^{[3]}$ Specifically, charge transfer at an externally polarizable ITIES (interface between two immiscible electrolyte solutions) can be driven and studied as a function of the applied Galvani potential difference between the two phases: $\Delta \phi_{\text {app }}=\phi^{\mathrm{w}}-\phi^{\text {org }}{ }_{{ }^{[4]}}$ Since the original four-electrode set-up with macrolTIES ${ }^{[4 c, 4 d]}$, the reduction of the interfacial area has been attempted primarily to minimize ohmic drop and capacitive effects Different strategies have been followed including the use of micro/nano-pipettes and holes ${ }^{[4--g]}$ and their arrays, ${ }^{[4 h]}$ microdroplet-modified electrodes ${ }^{[4 i-k]}$ and the scanning electrochemical microscope. ${ }^{[4]}$ They have collectively extended liquid|liquid electrochemistry, aiming for accurate measurements with highly resistive solutions as in biological membranes. Nevertheless, challenges persist related to the quantitative characterization and modelling of the interface dynamics and support electrostatic effects at pipettes, and to the complex behaviour observed at droplet-modified electrodes (Marangonitype effects, ohmic drop, intra-droplet diffusion,...).

Here, a novel and simple methodology is developed for the detection and analysis of individual micro- and nano-particles in solution and for the investigation of ion transfer processes accompanying fusion events. This extends the state-of-the-art

[a] Dr. E. Laborda, Prof. Dr. A. Molina, Ms V. F. Espín,

Prof. Dr. F. Martínez-Ortiz, Prof. Dr. J. García de la Torre Department of Physical Chemistry

Regional Campus of International Excellence "Campus Mare

Nostrum", University of Murcia, Murcia 30100 (Spain)

E-mail: elaborda@um.es

[b] Prof. Dr. R.G. Compton

Department of Chemistry, Physical \& Theoretical Chemistry Laboratory

Oxford University, South Parks Road, Oxford, OX1 3QZ (UK)

Supporting information for this article is given via a link at the end of the document. technique of impact experiments via the use of ionic probes (not redox ones) and liquid|liquid interfaces, which are notably reproducible and defect-free. Indeed, collision electrochemical methods have emerged as a simple and cost effective alternative for the study of "hard"[1] and "soft"[2] (such as emulsion droplets ${ }^{[2 a-}$ c], viruses ${ }^{[2 \mathrm{~d}-\mathrm{e}]}$, vesicles ${ }^{[2-\mathrm{g}]}$, micelles $^{[2 \mathrm{~h}]}$, liposomes $^{[2-\mathrm{i}]}$, organelles ${ }^{[20]}$, bacteria ${ }^{[2 p]}$, cells ${ }^{[2 q-s]}$ ) particles responding to the intense interest in stochastic processes and single-particle properties and to the need for understanding and monitoring these entities.

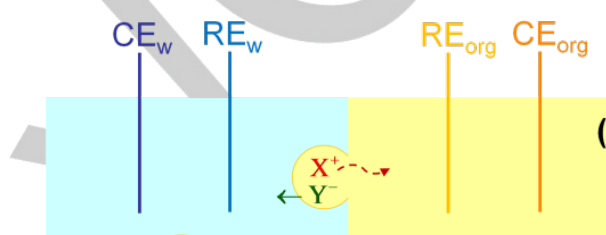

(A)

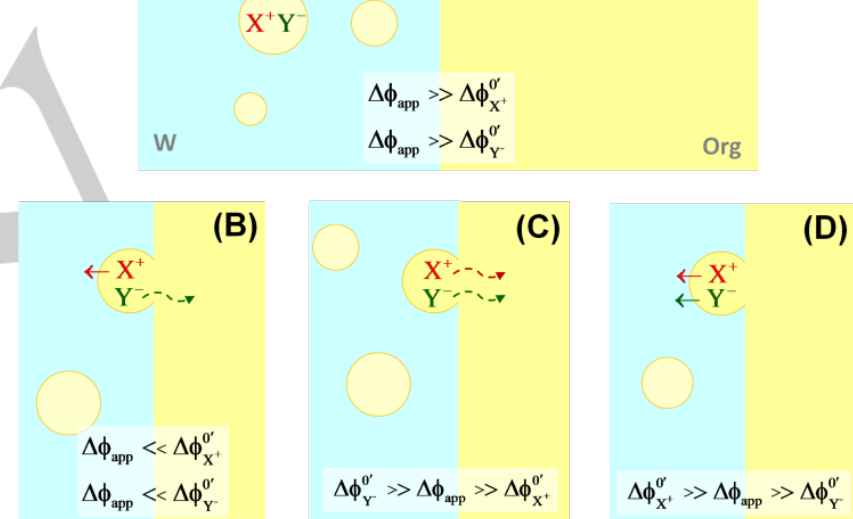

Scheme 1. Four different situations that can arise upon the fusion of an emulsion droplet with a polarized ITIES

Scheme 1 illustrates the physicochemical principle behind the novel approach based on registering the current-time signal of a polarized ITIES with the particles under study being dispersed in one of the phases. For example, consider oil droplets containing the dissolved salt $\mathrm{X}^{+} \mathrm{Y}^{-}$and diffusing freely in the aqueous phase. At some time, one of the droplets may collide and fuse with the polarized interface, with different situations being envisaged depending on the $\Delta \phi_{\text {app }}$-value and on the hydrophilicity of the ions (i.e., on their formal transfer potential, $\Delta \phi_{\mathrm{X}^{+}}^{0^{+}}$and $\left.\Delta \phi_{\mathrm{Y}^{-}}^{0^{\prime}}[3,5]\right)$. In those scenaria where there is net charge transfer between the two phases, a transient current flow is expected and the collision can be monitored. Thus, in case (A) where the applied potential difference is significantly more positive than the formal potentials of both ions, only the egress of anion $Y^{-}$to water is thermodynamically favorable, which would result in a current transient of positive sign (following the accepted convention). On the other hand, a negative current transient is expected in case (B) where the cation $\mathrm{X}^{+}$egresses to the water solution. In other words, the colliding droplet can be viewed as a tiny injection of the transferable ion in a (sub-)microscale region next to the polarized interface with such region behaving as an 'unsupported' micro- (or nano-) ITIES with just small currents resulting and hence minimal ohmic distortion effects. 
Following the above reasoning, DCE-in-W emulsions were prepared with DCE microdroplets containing the salt of a highly hydrophilic anion $\left(\mathrm{Y}^{-} \equiv \mathrm{Cl}^{-}\right)$and a very hydrophobic cation $\left(\mathrm{X}^{+} \equiv \mathrm{TDDA}^{+}\right)$: Emulsion 1. Both ions have a very negative formal potential such that the transfer of chloride to water is thermodynamically highly favorable at any $\Delta \phi_{\text {app }}$-value of the potential window available (Figure S1) unlike the egress of TDDA ${ }^{+}$ (case $(A)$ ).

Figure 1 shows the current-time response recorded after the injection of Emulsion 1 at different dilution factors (left) and at different $\Delta \phi_{\text {app }}$-values (right). As predicted, unidirectional positive current transients are clearly observed in the chronoamperograms in all the wide range of potentials examined. The transients are spike-like in shape (inset), similar to those observed in impact experiments ${ }^{[1,2]}$ and also to those associated with single exocytosis events. ${ }^{[6]}$ Thus, the spike shape can be informative about the dynamics of the corresponding fusion and charge transfer processes ${ }^{[6,7]}$ and it can also alert of possible distortions by the potentiostat electronics. ${ }^{[7]}$ The frequency of spikes scales with the concentration of droplets and no spikes appear in the absence of emulsion, discarding the inference of spontaneous emulsification ${ }^{[8]}$ in our system.

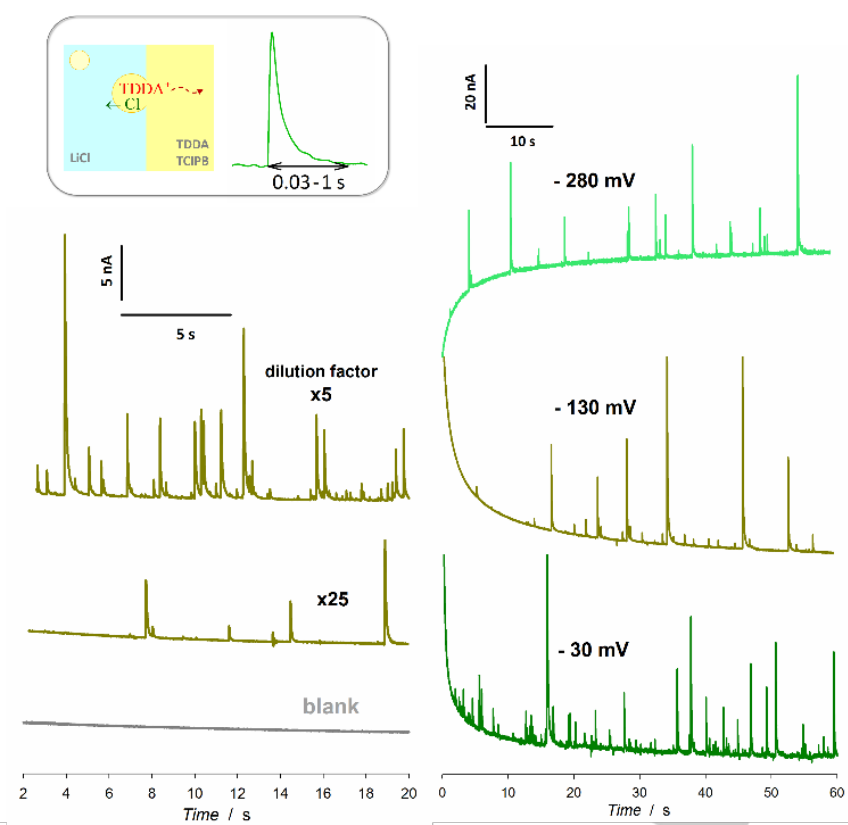

Figure 1. Chronoamperograms recorded with Cell I after injection of DCE-in-W emulsions with droplets bearing $0.35 \mathrm{M} \mathrm{TDDA}^{+} \mathrm{Cl}^{-}$(Emulsion1) at different dilution factors of the emulsion (the curve "blank" corresponds to the absence of emulsion droplets) (left) and at different applied Galvani potential difference (vs $\Delta \phi_{\mathrm{TMA}^{+}}^{0^{\prime}}$ ) (right)

Further confirmation of the nature of the current spikes was obtained by carrying out analogous experiments with emulsions where DCE droplets contained TMA ${ }^{+} \mathrm{TCIPB}^{-}$(Emulsion 2) or TDDA $^{+}$TCIPB $^{-}$(Emulsion 3). In the first case, the anion TCIPB' is very hydrophobic $\left(\Delta \phi_{\text {app }}<<\Delta \phi_{\mathrm{TClPB}^{-}}^{0^{\prime}}\right)$ whereas the formal transfer potential of the cation $\mathrm{TMA}^{+}$lies within our potential window (Figure $\mathrm{S} 1$ ). When $\Delta \phi_{\text {app }}$ is set at a value negative enough for $\mathrm{TMA}^{+}$to transfer to water, the experimental chronoamperograms recorded after injection of Emulsion 2 show current transients of similar shape to those observed with Emulsion 1 but of opposite sign (Figure 2, left). The spikes disappear when switching $\Delta \phi_{\text {app }}$ to a value notably more positive than $\Delta \phi_{\mathrm{TMA}^{+}}^{0^{+}}$where none of the ions of the salt dissolved in the droplets are expected to transfer to water (case (C) in Scheme 1). Regarding Emulsion 3, no ion transfer to water is favourable at any applied potential within the available potential window. Accordingly, no spikes are observed in the chronoamperograms recorded (Figure 2, right). Note that these results point out that no capacitive events associated with the collision of particles of micrometric size are observed with the current background signal.
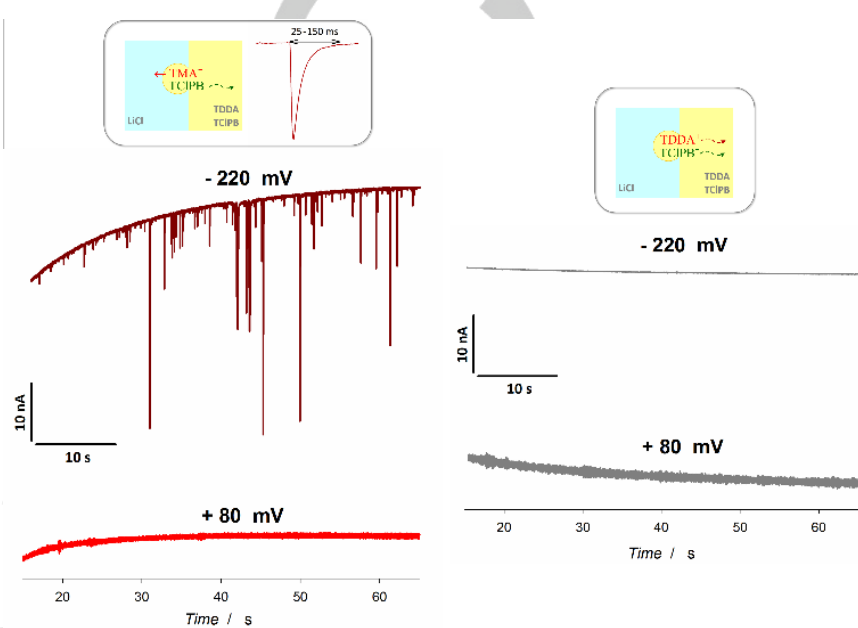

Figure 2. Chronoamperograms recorded with Cell I after injection of a DCE-inW emulsion with droplets bearing $0.025 \mathrm{M} \mathrm{TMA}^{+} \mathrm{TCIPB}^{-}$(Emulsion 2, left) and $0.35 \mathrm{M} \mathrm{TDDA}^{+} \mathrm{TCIPB}^{-}$(Emulsion 3, right) at two different $\Delta \phi_{\text {app }}$-values (vs $\left.\Delta \phi_{\mathrm{TMA}^{+}}^{0^{\prime}}\right)$

Finally, the capability of the new method to characterize individual particles and fusion events was examined. Under the assumption of exhaustive egress of the ionic probe, in case that each spike corresponds to the collision of a single droplet, then the charge under the spike $\left(Q_{\text {spike }}\right)$ allows for the estimation of the diameter of the spherical droplet, $\mathrm{d}$ :

$$
\mathrm{d}=2\left(\frac{3\left|\mathrm{Q}_{\text {spike }}\right|}{4 \pi \mathrm{F}\left|\mathrm{z}_{\mathrm{i}}\right| \mathrm{c}_{\mathrm{i}}}\right)^{1 / 3} ; \mathrm{i} \equiv \mathrm{X}^{+} \text {or } \mathrm{Y}^{-}
$$

where $F$ is the Faraday constant and $z_{i}$ and $c_{i}$ the charge and concentration of the transferred ion. Using Eq. (1), the droplet size distribution was obtained for samples of Emulsion 1 from experimental chronoamperograms recorded for $40 \mathrm{~min}$. The size distribution obtained electrochemically (grey bars in Figure 3) shows a droplet population in the region of diameters of 1 to $5 \mu \mathrm{m}$ with a mode value of ca. $1.8 \mu \mathrm{m}$ (ca. 24 femtoliter). The same emulsion sample was investigated simultaneously via dynamic light scattering (DLS, red line in Figure 3). Note that the two techniques have very different operating principles. Thus, the number of impacts 'seen' electrochemically reflects the diffusivity of the droplets so that inherently the number of smaller ones is 'overweighed', whereas intensity-based DLS distributions emphasize larger particles with higher intensity of light scattering than smaller ones. ${ }^{[9]}$ Considering these factors, the agreement between the two droplet size distributions can be taken as satisfactory (see also Figure S2), supporting the fact that each current spike is associated with the collision and fusion of an individual droplet. Note that this also means that the ionic content 
of individual droplets can be quantified with the present method. Thus, the number of ions $(\mathrm{N})$ contained in each colliding droplet under conditions of exhaustive egress (i.e., $\Delta \phi_{\text {app }}>\Delta \phi_{i}^{0^{\prime}}$ in the case of a transferable anion $i$ ) can be calculated directly from the charge of the corresponding spike:

$$
\mathrm{N}_{\mathrm{i}}=\frac{\left|\mathrm{Q}_{\text {spike }}\right|}{\left|\mathrm{z}_{\mathrm{i}}\right| \mathrm{e}} ; \mathrm{i} \equiv \mathrm{X}^{+} \text {or } \mathrm{Y}^{-}
$$

where $\mathrm{e}$ is the elementary charge $\left(; 1.6 \times 10^{-19} \mathrm{C}\right)$. For example, the charge values obtained in the study of Figure 3 ranged mainly between $0.02 \mathrm{nC}$ and $4 \mathrm{nC}$, which corresponds to $1.2 \times 10^{8}\left(2 \times 10^{-}\right.$ $\left.{ }^{16} \mathrm{~mol}\right)$ and $2.5 \times 10^{10}\left(4 \times 10^{-14} \mathrm{~mol}\right)$ chloride ions per DCE droplet, respectively.

In summary, current spikes of a stochastic nature associated with single fusion events at polarized liquid|liquid interfaces have been detected and analysed quantitatively, and the underlying ion-transfer process has been elucidated. The use of small size ITIES was essential to make the spikes visible and to minimize other sources of signal "instabilities". ${ }^{[8]}$ These results establish a new framework for single-entity electrochemistry with potential direct application to the study of the concentration, microstructure and behaviour of emulsions, liposomes, vesicles and liquid microreactors. Strategies based on particles adsorbing at the interface ${ }^{[10]}$ and blocking some ion-transfer signal[2a] can extend the approach to 'hard' particles. Also, further reduction of the polarized interface will allow for the investigation of nanometric entities.

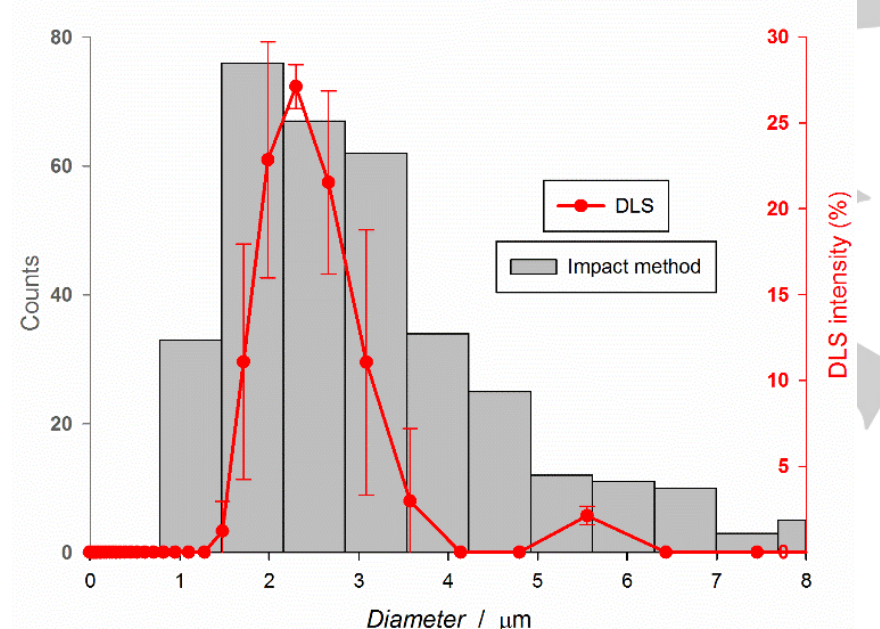

Figure 3. Droplet size distributions obtained electrochemically and by DLS of DCE-in-water emulsions with droplets bearing $0.35 \mathrm{M} \mathrm{TDDA}^{+} \mathrm{Cl}^{-}$(Emulsion1). Error bars represent the standard deviations obtained from 5 consecutive mesuraments within a period of $40 \mathrm{~min}$

Apart from the direct application of the method to the sizing of microparticles and the determination of their ionic content, the novel approach has also likely impact for the investigation of ion transfer processes across liquid|liquid interfaces at the (sub)microscale, without requiring sophisticated instrumentation and eliminating masking or distorting effects of alternative strategies. Thus, studies of the potential dependence of the spike features (charge and shape) can give a more comprehensive view of the open questions about the kinetics and mechanisms ${ }^{[11]}$ of ion transfers. Also, this will enable the determination of the lipophilicity of ionic compounds even with respect to media of very low dielectric constant. Moreover, with the use of phospholipidmodified ITIES ${ }^{[12]}$, understanding can be gained about charge transfer and uptake-release processes of biological interest and about interactions of biomembranes with (sub)micrometric entities. Quantitative applications call first for the identification and modelling of the governing mass transport, electrical and thermodynamic forces in these systems and of the dynamics of the fusion.

\section{Experimental Section}

Tetradodecylammonium tetrakis-(4-chlorophenyl)borate (TDDA TCIPB), tetradodecylammonium chloride (TDDA $\mathrm{Cl}$ ), tetramethylammonium chloride (TMACl), potassium tetrakis-(4-chlorophenyl)borate (K TCIPB), lithium chloride ( $\mathrm{LiCl}, 8 \mathrm{M}$ solution) and 1,2-dichloroethane (DCE) were obtained from Sigma Aldrich. Tetramethylammonium tetrakis-(4chlorophenyl)borate (TMATCIPB) was prepared by metathesis of TMACl with KTCIPB. All aqueous solutions were prepared with deionized Milli-Q water.

DCE-in-water emulsions were prepared by sonication for 3 min of 30-50 $\mu \mathrm{L}$ of the corresponding DCE solution and $2 \mathrm{~mL}$ of $0.2 \mathrm{M} \mathrm{LiCl}$ water solution. Droplet diameters in the resulting emulsions were found to lie in the range of a few microns according to DLS measurements. For the experiments, emulsions were diluted (x10 unless otherwise indicated) in $0.2 \mathrm{M} \mathrm{LiCl}$ solution.

Electrochemical measurements were performed with a BioLogic SP-200 potentiostat and a 4-electrode set-up housed inside a grounded Faraday cage. The current response was sampled every $1 \mathrm{~ms}$, which provides welldefined spikes (insets in Figures 1 and 2). Two silver wires acted as quasireference electrodes and two platinum wires as counter electrodes. The scheme of the cell is:

\section{$\mathrm{Ag} \mid$ 0.02M TDDA TCIPB (DCE) | 0.2M LiCl (W) | Ag}

(Cell I)

Fresh liquid|liquid interfaces were created for each assay with $0.15 \mathrm{ml}$ of DCE and water solutions in a cylindrical channel of a homemade U-tube. The interfacial area as obtained by chronoamperometry was $0.24 \pm 0.02 \mathrm{~mm}^{2}$. Control experiments confirmed that mutual saturation of the solvents did not influence the behaviours reported and also that the liquid|liquid interfaces were stable during and after impact experiments. Distortion of the signal by the electronics could be discarded after observing no effects on the spike features when varying the response time of the potentiostat within the upper range of bandwidth frequencies available. Moreover, the present analysis only uses the total charge, not the spike shape, and the former is known to be conserved even if there was a little distortion. ${ }^{[7]}$

The hydrodynamic size of the emulsion droplets was measured by DLS using a Malvern Zetasizer Nano ZS. Data acquisition times for accurate intensity particle size distributions were set to 300 seconds according to the analysis of polystyrene latex standards (Sigma-Aldrich) of size similar to the emulsion droplets (Figure S3).

\section{Acknowledgements}

$\mathrm{EL}, \mathrm{AM}$ and FM-O thank the financial support of the Fundación Séneca de la Región de Murcia (18968/JLI/13 and 19887/GERM/15) and the MINECO (Spanish government, Project CTQ2015-65243-P and Juan de la Cierva fellowship). VFE thanks the predoctoral fellowship from MINECO Grant. JGT 
thanks the funding received from the Fundación Séneca (19353/PI/14).

Keywords: single fusion events $\cdot$ electrochemistry $\bullet$ polarized liquid|liquid interfaces $\bullet$ impact experiments $\bullet$ microparticles

[1] a) A.J. Bard, A. Boika, S.J. Kwon, J. Hui Park, S.N. Thorgaard, in Nanoelectrochemistry (Eds.: M.V. Mirkin, S. Amemiya), CRC Press, Boca Raton, 2015, pp. 241-292; b) H.S. Toh, R.G. Compton, in Electrochemical Strategies in Detection Science (Ed.: D.W.M. Arrigan), RSC, Cambridge, 2016, pp. 170-204

[2] a) B-K. Kim, A. Boika, J. Kim, J.E. Dick, A.J. Bard, J. Am. Chem. Soc. 2014 136, 4849-4852; b) H. Deng, J.E. Dick, S. Kummer, U. Kragl, S.H. Strauss, A.J. Bard, Anal. Chem. 2016, 88, 7754-7761; c) W. Cheng, R.G. Compton, Angew. Chem. Int. Ed. 2016, 55, 2545 -2549; d) J.E. Dick, A.T. Hilterbrand, A. Boika, J.W. Upton, A.J. Bard, Proc. Natl. Acad. Sci. U.S.A. 2015, 112, 5303-5308; e) L. Sepunaru, B.J. Plowman, S.V. Sokolov, N.P. Young, R.G. Compton, Chem. Sci. 2016, 7, 3892-3899; f) J. Dunevall, H. Fathali, N. Najafinobar, J. Lovric, J. Wigström, A-S. Cans, A.G. Ewing, J. Am. Chem. Soc. 2015, 137, 4344-4346; g) X. Li, S. Majdi, J. Dunevall, H. Fathali, A.G. Ewing, Angew. Chem. Int. Ed. 2015 54,11978-11982; h) H.S. Toh, R.G. Compton, Chem. Sci. 2015, 6, 50535058; i) D. Hellberg, F. Scholz, F. Schauer, W. Weitschies, Electrochem. Commun. 2002, 4, 305-309; j) D. Hellberg, F. Scholz, F. Schubert, M. Lovric, D. Omanovic, V.A. Hernández, R. Thede, J. Phys. Chem. B 2005 109, 14715-14726; k) V.A. Hernández, F. Scholz, Langmuir 2006, 22 10723-10731; I) V.A. Hernández, F. Scholz, Bioelectrochem. 2008, 74 149-156; m) V.A. Hernández, M. Hermes, A. Milchev, F. Scholz, J. Solid State Electrochem. 2009, 13, 639-649; n) W. Cheng, R.G. Compton Angew. Chem. Int. Ed. 2014, 53, 13928 -13930; o) M. Hermes, F. Scholz, C. Härdtner, R. Walther, L. Schild, C. Wolke, U. Lendeckel, Angew. Chem. 2011, 123, $7004-7007$; p) L. Sepunaru, K. Tschulik, C. Batchelor-McAuley, R. Gavish, R.G. Compton, Biomater. Sci. 2015, 3, 816-820; q) L. Sepunaru, S.V. Sokolov, J. Holter, N.P. Young, R.G. Compton, Angew. Chem. 2016, 128, 9920-9923; r) J.E. Dick, Chem. Commun. 2016, 52, 10906-10909; s) J.J. Gooding, Angew. Chem. Int. Ed. 2016, 55, 12956-12958

[3] a) H.H. Girault, in Developments in Electrochemistry: Science Inspired by Martin Fleischmann (Eds.: D. Pletcher, Z-Q. Tian, D.E. Williams), Wiley,
Chichester, 2014, pp. 295-308; b) A. Molina, C. Serna, J.A. Ortuño, E. Torralba, Annu. Rep. Prog. Chem., Sect. C: Phys. Chem. 2012, 108 126-176; c) F. Scholz, Annu. Rep. Prog. Chem., Sect. C 2006, 102, 4370; d) Z. Samec, Pure and Applied Chemistry 2004, 76, 2147-2180

[4] a) C. Gavach, T. Mlodnicka, J. Guastalla, C.R. Acad. Sci. C 1968, 266, 11961199; b) J. Koryta, M. Březina, P. Vanýsek, J. Electroanal. Chem. 1977, 75, 211-228; c) C. Gavach, F. Henry J. Electroanal. Chem. 1974, 54 361-370; d) Z. Samec, V. Mareček, J. Weber. J. Electroanal. Chem. 1979, 100, 841-852; e) G. Taylor, H. H. Girault, J. Electroanal. Chem. 1986, 208, 179-183; f) S. Liu , Q. Li, Y. Shao, Chem. Soc. Rev. 2011, 40, 22362253; g) M.V. Mirkin, in Nanoelectrochemistry (Eds.: M.V. Mirkin, S. Amemiya), CRC Press, Boca Raton, 2015, pp. 539-572; h) D.W.M. Arrigan, Y. Liu Annu Rev. Anal. Chem. 2016, 9, 145-61; i) F. Marken, R.D. Webster, S.D. Bull, S.G. Davies J. Electroanal. Chem. 1997, 437 209-218; j) F. Scholz, S. Komorsky-Lovric, M. Lovric Electrochem. Commun. 2000, 2, 112-118; k) F. Scholz, U. Schröder, R. Gulaboski, A. Doménech-Carbó Electrochemistry of Immobilized Particles and Droplets, Springer, Cham, 2014; I) C. Wei, A. J. Bard, M. V. Mirkin. J. Phys. Chem. 1995, 99, 16033-16042

[5] http://sbsrv7.epfl.ch/instituts/isic/lepa/cgi/DB/InterrDB.pl

[6] F. Lemaître, M.G. Collignon, C. Amatore, Electrochim. Acta 2014, 140, 457466

[7] E. Kätelhön, E.E.L. Tanner, C. Batchelor-McAuley, R.G. Compton, Electrochim. Acta 2016, 199, 297-304

[8] a) T. Kakiuchi, Electrochem. Commun. 2000, 2, 317-321; b) T. Kakiuchi, in Interfacial Nanochemistry (Eds.: H. Watarai, N. Teramae, T. Sawada), Kluwer Acad./Plenum Publishers, New york, 2005, pp.155-170; c) Y. Kitazumi, T. Kakiuchi, J. Phys.: Condens. Matter 2007, 19, 375104

[9] M. Collado-González, V.F. Espín, M.G. Montalbán, R. Pamies, J.G.H. Cifre, F.G.D. Baños, G. Víllora, J.G. de la Torre, J. Nanopart. Res. 2015, 17, 268

[10] a) B. Su, J-P. Abid, D.J. Fermín, H.H. Girault, H. Hoffmannova, P. Krtil, Z. Samec, J. Am. Chem. Soc. 2004, 126, 915-919; b) P.S. Toth, A.N.J. Rodgers, A.K. Rabiu, D. Ibañez, J.X. Yang, A. Colina, R.A.W. Dryfe, J. Mater. Chem. A 2016, 4, 7365-7371

[11] a) Z. Samec, Electrochim. Acta 2012, 84, 21-28; b) A. Trojánek, V. Marecek, Z. Samec, Electrochim. Acta 2015, 180, 366-372; c) N. Kikkawa, L. Wang, A. Morita J. Chem. Phys. 2016, 145, 014702

[12] H.A. Santos, V. García-Morales, C.M. Pereira, ChemPhysChem 2010, 11, 28-41 


\section{COMMUNICATION}

The collision and fusion of individual liquid microparticles with polarized liquid|liquid interfaces are detected and analyzed via electrochemical impact experiments with ionic probes. The method developed opens a novel approach for tracking micro- and nano-entities in solution and for getting insights into their interactions with soft biomimetic interfaces as well as into the kinetics and mechanisms of interfacial ion transfers.
Eduardo Laborda*, Angela Molina, Vanesa Fernández Espín, Francisco Martínez-Ortiz, José García de la Torre, Richard G. Compton

Single Fusion Events at Polarized Liquid|Liquid Interfaces 